\title{
3D Multi-gate Transistors: Concept, Operation, and
} Fabrication

\author{
Nader Shehata ${ }^{1,2}$, Abdel-Rahman Gaber ${ }^{3}$, Ahmed Naguib $^{3}$, Ayman E. Selmy ${ }^{4}$, Hossam Hassan ${ }^{3}$, Ibrahim Shoeer ${ }^{3}$, \\ Omar Ahmadien ${ }^{3}$ and Rewan Nabeel ${ }^{3}$ \\ 1. Department of Engineering Mathematics and Physics, Faculty of Engineering, Alexandria University, Alexandria 21544, Egypt \\ 2. CSNP (Center of Smart Nanotechnology and Photonics), Smart CI Research Center, Alexandria University, Alexandria 21544, \\ Egypt \\ 3. Department of Electrical Engineering, Faculty of Engineering, Alexandria University, Alexandria 21544, Egypt \\ 4. School of Sciences and Engineering, the American University in Cairo, P.O. Box 74 New Cairo 11835, Egypt
}

\begin{abstract}
The multi-gate transistors such as Fin-FETs, Tri-gate FETs, and Gate-all-around (GAA) FETs are remarkable breakthrough in the electronic industry. 3D Transistor is taking the place of the conventional 2D planar transistor for many reasons. 3D transistors afford more scalability, energy efficient performance than planar transistors and increase the control on the channel region to reduce the short channel effect, which enables us to extend Moore's law to further extent. In this paper, we will present a review about their structure, operation, types and fabrication.
\end{abstract}

Key words: Fin-FET, transistor fabrication.

\section{Introduction}

Since the 1970's, the electronics industry has been subjected to Moore's law. According to Moore, every eighteen months approximately the size of the transistor gets reduced to half of its original size. So, the number of transistors on the integrated circuit duplicates. Basically, a transistor is a three-terminal semiconductor device used to amplify and/or switch electronic signals and power. Either current or voltage between two of the terminals can be controlled by applying an electric current or voltage to the third terminal. A transistor can amplify a signal where the controlled "output" signal can be higher than the controlling "input" signal. Today, some transistors are packaged individually, but many more are integrated in electronic chips integrated circuits, IC's. The transistor is the fundamental building block of modern electronic devices $[1,2]$.

Corresponding author: Nader Shehata, assistant professor, Ph.D., research field: nanoelectronics. E-mail: nader.shehata@alexu.edu.eg.
Transistors have many different types: the planar transistors, and the non-planar transistors, including the Multi-gate transistors, called Fin-FET's, and 3D transistors. These transistors employ a single gate stacked on top of two vertical gates allowing for essentially almost three times the surface area for electrons to travel [1]. FinFET transistors reduce leakage and consume less power than planar transistors. The additional gate control enables as much transistor current flowing as possible when the transistor is in the "on" state (for performance), and nearly zero possible when it is in the "off" state (to minimize power), and enables the transistor to switch very quickly between the two states [3]. The acronym 3D here does not refer to images but to physical and novelty of a design and manufacturing technique, just as you can store more cars in a multi-storey garage than in a flat parking lot, more circuitry can be packed in three dimensions, but of course with a trade off with complex design. [1] 


\section{Structure}

Every Silicon wafer consists of hundreds of chips, each chip consists of millions of transistors, and every transistor consists of two regions called "source" and "drain". In Fig. 1, MOSFET (metal oxide semi-conductor field effect transistor) device is illustrated, in NMOS the two regions are n-type, and separated by a p-type region called the substrate. PMOS devices are constructed in a different way where the two regions are p-type and the substrate is of n-type.

A thin layer of insulator material covers the region between the source and the drain, over it, there is a metal electrode, from which the word "Metal" came in the name "MOSFET" (metal oxide semi-conductor field effect transistor), and it is named the "gate". When a voltage is applied at the gate terminal, a channel of electrons is formed between the source and the drain, so this device acts as a voltage controlled current source [2-4].

In tri-gate transistors, there are conducting channels on the three sides of the vertical fin. It has one gate electrode on the top and two gate electrodes on the sides, so the control of the gate increases in a way that in the "on" state current passes as much as possible, and in the "off" state current passes is almost zero. This makes the switching velocity between the two states higher, which results in a better performance for the transistor [1]. In addition, the performance advantage can be provided by the control of the effective width of the conducting channel [5]. This width is greater in $3 \mathrm{D}$ tri-gate transistor because it increases in the third dimension of this structure, which is the main difference between 2D (Planar) and 3D transistors without increasing the overall footprint of the transistor as shown in Fig. 2.

By controlling the effective channel length, the short channel effect can be controlled in 3D transistors and since the fins are vertical, high packing density can be achieved by packing transistors close together, and this will result in higher performance [1-3].
Through keeping the ratio between the effective channel length and fin width bigger than 0.5 , the channel doping will decrease, impurity in channel will decrease, so volume inversion effect will increase and higher performance will be achieved specially in lower bias region [3].

Multi-gate transistors can be fabricated on SOI substrate or standard bulk substrate. Fig. 3 shows the different ways in which the gate electrode can be wrapped around the channel region of a transistor (a) SOI Double-gate FETs: we can notice the "hard mask", which is a thick dielectric that prevents the formation of an inversion channel at the top of the silicon "fin". Gate control is exerted on the channel from the sides of the device; (b) SOI triple-gate (tri-gate) MOSFETS: Gate control is exerted on the channel from three sides of the device (the top, as well as the left and right sides); (c) SOI -gate MOSFET: Gate control is improved over the tri-gate MOSFET shown in (b) because the electric field from the sides

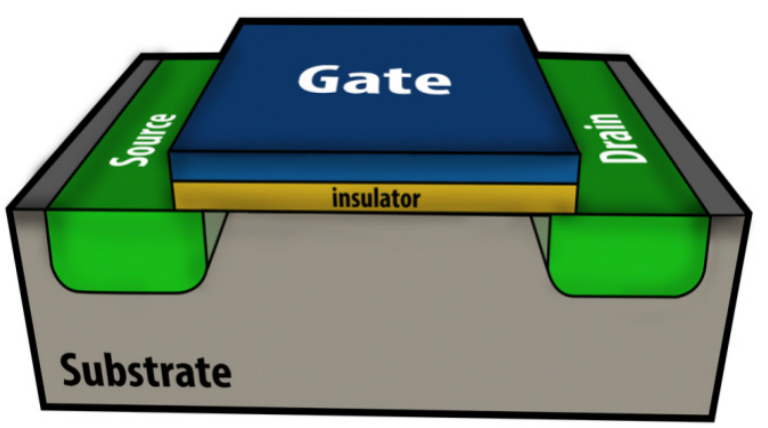

Fig. 1 Schematic diagram of ordinary MOSFET.
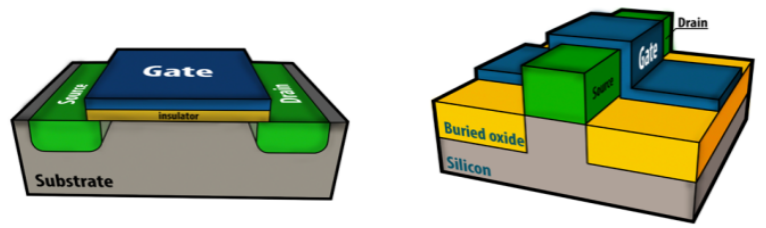

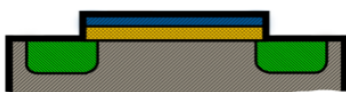

Planar MOSFET

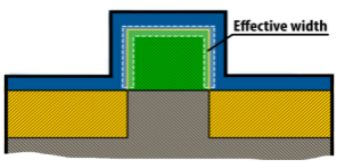

Tri-Gate MOSFET
Fig. 2 Channel effective width in planar and multi-gate transistors. 

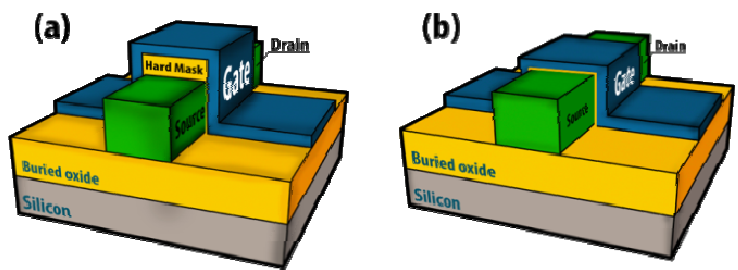

(c)

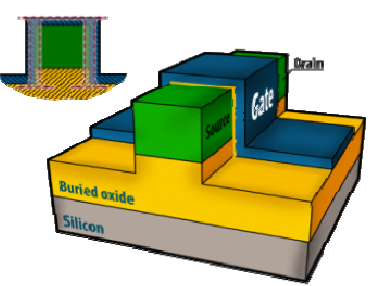

(e)

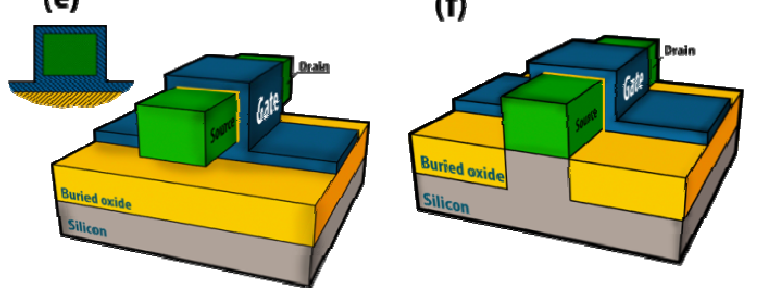

Fig. 3 Different types of multi-gate transistors.

of the gate exerts some control on the bottom side of the channel; (d) SOI -gate MOSFET: Gate control of the bottom of the channel region is better than in the -gate MOSFET. The names -gate and -gate reflect the shape of the gates; (e) SOI gate-all-around "GAA" MOSFET. Gate control is exerted on the channel from all four sides of the device. In this case, there is no buried oxide underneath the silicon channel. All of the above devices were made using SOI (silicon-on-insulator) substrates, SOI substrates consist of a thin single-crystal silicon layer sitting on top of an insulator, usually silicon dioxide. Multi-gate FETs can also be made with bulk silicon wafers instead of an SOI substrate, in this case the channel is connected to the substrate silicon without insulator between them, as shown in Fig. 3f [1-5].

\subsection{Double Gate and Tri-gate}

Now we get to compare the characteristics of the double gate and the tri-gate SOI Fin-FET. Talking about the threshold extracted using the constant current method [6, 7]; it was found that the threshold is reduced in the SOI Fin-FET by reducing the $t_{\mathrm{ox}}$ while in the bulk it increases with the reduction of the $t_{\mathrm{ox}}$. Considering the width of the fin in the threshold, voltage decreases with the increase of width in both the double gate and the tri-gate SOI Fin-FET, but with less sensitivity in the latter due to the third gate that compromises the loss of control of the other two gates. Also we can find that the tri-gate is better than the double gate SOI Fin-FET in the voltage threshold, and also in the sub-threshold swing where the tri-gate has proved to be more effective and reliable [7].

Considering the DIBL (drain-induced barrier lowering) which is caused by the travelling of the depletion region from the drain to the channel; we can find that the DIBL is lower in the tri-gate bulk compared to double gate SOI Fin-FET. Another parameter affecting the DIBL is the width. When it increases, the DIBL also increases with more sensitivity in double gate SOI Fin-FET than that in tri-gate bulk. One more element is the channel length which when increased; the effect of the electric field washes out so the DIBL decreases a bit. This happens in both structures but the tri-gate shows much less values of DIBL in smaller channel lengths [7].

The last parameter of comparison in this section is the temperature. It was found that the peak temperature was lower in the bulk reaching $426 \mathrm{~K}$ while $448.5 \mathrm{~K}$ in the double gate [7]. Maybe this happens due to the opening under the channel in the bulk [8]. Now we can get to the conclusion that tri-gate has an upper hand at all the points of comparison mentioned and it is the key to future reliable designs [7].

\subsection{Pi Gate and Omega Gate}

$\Pi$-gate and $\Omega$-gate are triple gate devices, with an extended gate electrode, which reduces the short-channel effects and increases the current drive. This extension blocks the drain electric field lines from reaching the channel by forming an opposing virtual gate electrode. Because of terminating the drain electric field, the short-channel effect will be 
reduced, as the extension operates as a virtual back gate. This termination almost prevents the occurrence of DIBL and eventually the punch through effect [9]. An economic advantage of the Pi gate is that it simplifies the fabrication process of the complicated GAA manufacturing with almost the same specifications and nearly the same short-channel characteristics [10].

\section{Operation}

To understand the operation of any type of the multi-gate transistors we must first have a brief introduction about the operation of traditional planar MOSFET

\subsection{Planar MOSFET}

Planar MOSFET consists of gate, insulator and substrate (Fig. 1). If we applied positive potential on the gate, it repels the positive charges (holes in NMOS) of the p-type substrate, so this region is depleted of carriers and remains with the fixed negative ions. After we increase the voltage, negative carriers (electrons in NMOS) are attracted from this substrate to the region underneath the insulator material, forming a channel (channel inversion) between the source and the drain. If a voltage is applied between the source and the drain, current flows. The voltage $V_{D}$ controls the amount of this current [2-4].

To operate this device, two potentials must be applied, one at the gate node $\left(V_{G}\right)$ and another one at the drain node $\left(V_{D}\right)$. If $V_{G}<V_{T H}$ (where $V_{T H}$ is the threshold voltage which is the least voltage needed to create a channel), then no channel exists and the device is off. In this case $I_{D}=0$ regardless of the value of $V_{D}$. When $V_{G}>V_{T H}$, then $I_{D}>0$ (Figs. 4a-b) [2].

If $V_{D}$ remains higher than $V_{S}$, then the voltage increases as we go from the source to the drain at each point away along the channel with respect to ground. As the gate voltage is constant, the potential difference between the gate and the insulator interface decreases along the $\mathrm{x}$-axis as we approach the drain, so does the density of the electrons in the channel.

Therefore, if $V_{G}-V_{D} \leq V_{T H}$, then the channel stops near the drain and this is called "Pinched off", but the device still conducts due to the field effect that the electrons are subjected to in the depletion region around the drain junction, so they reach the drain terminal (Fig. 4c) [2].

Equation $Q=C V$ states that, to achieve a strong control of $Q$ by $V$, the value of $C$ must be maximized. One of the ways to achieve that is by reducing the thickness of the dielectric layer separating the two plates, the capacitance between two plates is given by $(\varepsilon . A) / t$ where " $\varepsilon$ " is the dielectric constant-permittivity, " $A$ " is the area of each plate, and " $t$ " is the dielectric thickness. The main goal here is to lower the thickness " $t$ " to increase the capacitance, and the accelerated advancement in silicon fabrication technology makes that goal easier [2].

As the thickness of the oxide increases, the capacitance between the gate and the silicon substrate decreases. Thus, from $Q=C V$, we note that a given voltage results in less charge on the gate and hence a lower electron density in the channel. Therefore, the device suffers from a higher on resistance, producing less drain current for a given gate and drain voltages. For this reason, the semiconductor industry has continued to reduce the gate oxide thickness [2].

\subsection{Multi-gate MOSFET}

The operation of multi-gate FETs shares the same

(a)

(b)
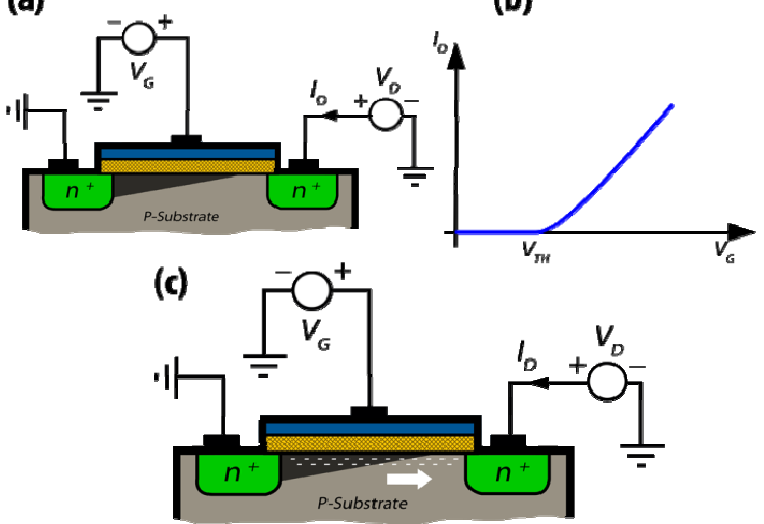

Fig. 4 Operation of planar MOSFET. 
idea as planar MOSFET, as we can consider it an advanced version of traditional MOSFET, but minimization of the transistor has some drawbacks, which affect the functionality of the transistor. Gate leakage current, tunneling leakage of carriers at high doping levels, rising power dissipation and short channel effect are some examples to these drawbacks as a result of the minimization process.

\subsubsection{Short Channel Effects}

Short channel effect is the most important drawback. It results from some consequences: the presence of a depletion region between the source and the drain results in the presence of an electric field flowing in the depletion region. This depletion region is created by the source and drain junctions occurring to shorten the effective channel length. This electric field affects the carriers flowing in the channel decreasing the gate control on them. The gate control is also influenced by the drain voltage and the distance between the source and the drain, as the drain voltage increases the effect of the electric field increases [1-5]. The main consequences of Short Channel effect as follows.

Velocity saturation: As we go smaller in size, the size of all parts in the transistor decreases including the length of the channel, which increases the electric field due to the inversely proportional relation of the length and the electric field $E=-\mathrm{d} v / \mathrm{d} x$. When the electric field increases the velocity of carriers increases according to the relation between electric field and charged particles $V=\mu^{*} E$, but this velocity will not increase infinitely, and will actually stop increasing at a specific value which is called (saturation velocity) because of increasing the rate of collision between carriers. This effect in velocity will result in channel mobility degradation, which will affect the flow of current in the channel region decreasing the performance of the transistor [1-11].

Punch through: It is the merging between the depletion regions of the drain and the source into a single depletion region [12]. The field flowing through the depletion region affects the current passing through the channel and the drain voltage becomes the dominant voltage affecting the current. Punch through can be minimized by several methods, the first method is decreasing the thickness of the oxide, which provides more control for the gate voltage over the channel current, the second method is increasing the substrate doping, which prevents the merging of the depletion regions of the source and the drain, and other method is using shallower junctions which confines the depletion regions making the linkage more difficult [11].

Surface scattering: It is the degradation in the surface mobility of the channel carriers to half of that of the bulk region due to the vertical component of the electric field causing the collision with the interfacing surface between the channel and the bulk region [11].

3.2.2 Relation between Threshold Voltage and Channel Length

The threshold voltage is the least gate-to-source voltage capable of creating a conducting channel by attracting bulk charges that causes channel inversion [2]. In small dimensioned MOSFETs, the actual threshold voltage is less than that calculated value estimated by the standard equation, because the equations assume that all the bulk charges attracted to generate the channel are due to the gate voltage only, while some charges are attracted due the electric field generated by the PN junction charges at the source and the drain [11].

Another factor affecting the threshold voltage is hot carriers degradation which means that there are high energy electrons penetrating the oxide and getting trapped and accumulated there, decreasing the device characteristics by increasing the channel field thus decreasing the threshold voltage [11].

It was found that in tri-gate NMOS $22 \mathrm{~nm}$ technology that the threshold voltage is less than that of tri-gate NMOS $32 \mathrm{~nm}$ technology, it also has a faster increase with the drain voltage because it provides the gate with more control [11]. 


\subsubsection{Electrostatic Control of Channel}

A MOSFET device is free of short-channel effects if the gate length is at least 4 to 6 times larger than the natural length of the device. $\lambda$ is the natural length that refers to the potential distribution of the whole structure [5]. It represents the field lines penetration from the source and the drain in the channel region. From Poisson's equation, the natural length is given by:

$$
\lambda_{n}=\sqrt{\frac{\varepsilon_{s i}}{n \varepsilon_{o x}}} t_{o x} t_{s i}
$$

where, " $n$ " is the equivalent number of gates $(n=2$ for a double gate device, $n=3$ for a tri-gate device, $n=4$ for a GAA transistor), $\varepsilon_{s i}$ is the electrical permittivity of the channel, $\varepsilon_{o x}$ is the electrical permittivity of the gate dielectric, $t_{s i}$ is the film thickness and $t_{o x}$ is the gate dielectric thickness $[13,14]$.

\subsubsection{Quantum Effects}

The continuous decrease of the device dimensions leads to the appearance of Quantum effects, like Quantum confinement, volume inversion, and current oscillation, which introduces new challenges in device physics and modeling [15]. Carriers in narrow triple or quadruple FETs are confined in two directions (y and $\mathrm{z}$ ), which are perpendicular to the direction of carriers flow $x$, as shown in Fig. 5. We can neglect the confinement effects if the wire was large enough, but in smaller section FETs, this confinement causes electron distribution that differs from the predicted one from classical theory [16].

In the planar or wider multi-gate FETs, inversion layers are localized at the surface of the silicon film, but in narrower wires it can be found in the center of the film. This causes the phenomenon of "volume inversion" which affects the mobility and threshold voltage behaviors [5-16]. Volume inversion is a phenomenon that happens to very thin film multi-gate SOI MOSFETs, in this case the inversion carriers are not confined near the surface of the film, but at its center [17]. The carriers concentration profile at $V_{G}=$ $V_{T H}+0.7$ in tri-gate SOI transistors in strong inversion for different square cross sections $[15,16]$.

Confinement raises the formation of sub-bands. As the gate voltage increases, the electron concentration increases, so a larger number of sub-bands become populated. All these factors lead to inter-sub-band scattering between electrons from different energy sub-bands [15]. Inter-sub-band scattering increases with each new sub-band that becomes populated, which results in mobility reduction. Therefore, oscillations of the drain current occur, when the gate voltage is increased. Current oscillations can be observed as a function of gate voltage, when the drain

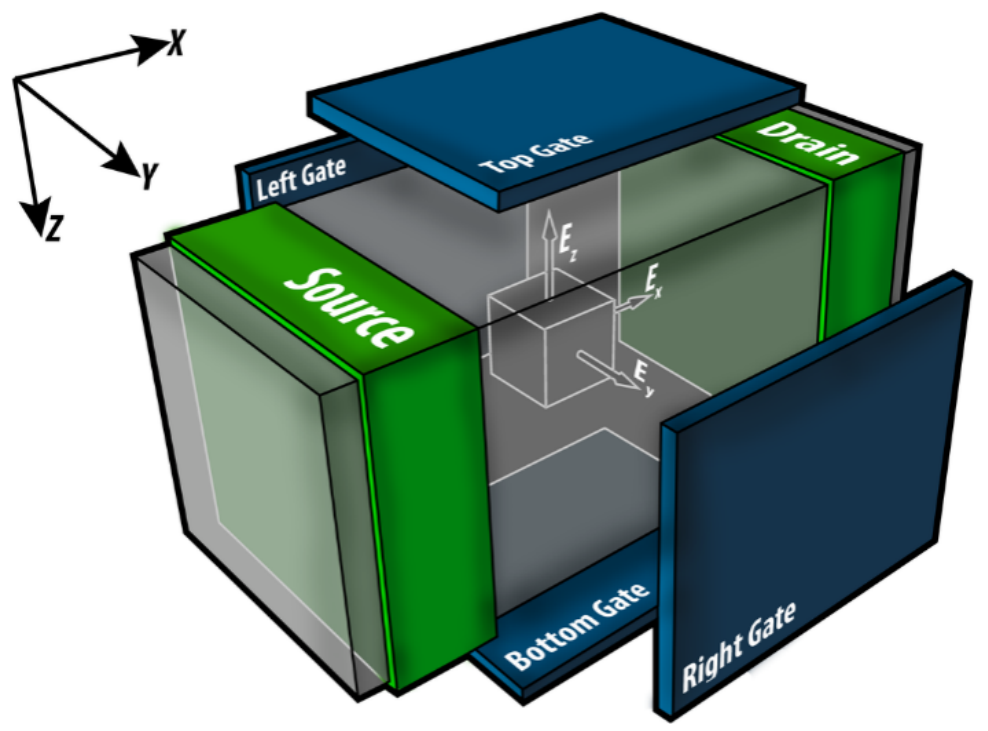

Fig. 5 Element volume in a channel region. 
voltage is not significantly larger than the energy separation between sub-bands, which is expressed by " $\Delta E$ " divided by the electron charge " $q$ " [15-19].

\subsubsection{Tri-gate Impact on I-V Characteristics}

For large dimensions (as in $90 \mathrm{~nm}$ technology), the ratio between the drain to source current and the simple gate transistor value is 1.1:1, while for smaller dimensions (as in 22.5 technology) the ratio between them is $1.44: 1$ [20, 21].

A new revolutionary speed and gain performance introduced at FPGAs based on $22 \mathrm{~nm} 3 \mathrm{D}$ tri-gate technology. These FPGAs will operate $37 \%$ faster at low power compared to $32 \mathrm{~nm}$ planar FPGAs, and will save more than $50 \%$ of the power consumed, which makes them suitable in handheld devices as they can operate at less energy, but would still have poor gate delay at low voltage [3]. That's how the problem of leakage current flowing through the channel when the gate voltage equals zero seems to be solved; as lower amount of it flows through the channel of the tri-gate transistors than that flowing in planar transistors. The dynamic power is reduced with reducing the transistor channel dimensions, which means that the technology of 3D tri-gate improves the control over static and active power dissipations of FPGAs [3].

\section{Fabrication}

\subsection{Fabrication Materials and Technology}

A metal gate, in the context of a lateral metal-oxidesemiconductor MOS stack, is just that the gate material is made from a metal. The primary criterion for the gate material is that it is a good conductor. For decades the industry has been moving away from metal (most typically aluminum evaporated in a vacuum chamber onto the wafer surface) being used as the gate material in the MOS stack, due to fabrication complications [22]. A material called poly-silicon was used to replace aluminum because the threshold voltage and the drain to source on-current is modified by the work function difference between the gate material and channel material. Because poly-silicon is a semiconductor, its work function can be modulated by adjusting the type and level of doping [23], the silicon dioxide $-\mathrm{SiO}_{2}$ interface has been well studied and is known to have relatively few defects. By contrast, many metal-insulator interfaces contain significant levels of defects, which can lead to Fermi level pinning, charging, or other phenomena that ultimately degrade the device performance, fabrication processes where the initial doping requires very high temperature annealing. Metal gates would melt under such conditions whereas poly-silicon would not. Using poly-silicon paved the way for a one-step process of etching the gates compared elaborating multi-steps that we see today in metal-gate processes [24].

While poly-silicon gates have been the de facto standard for the last twenty years, however, they do have some problems, which have led to their likely future replacement by metal gates. Poly-silicon is not a great conductor, which reduces the signal propagation speed through the material. The resistivity can be lowered by increasing the level of doping, but even highly doped poly-silicon is not as conductive as most metals. To improve conductivity further, sometimes a high-temperature metal such as tungsten, titanium, cobalt, and more recently nickel is alloyed with the top layers of the poly-silicon. Such a blended material is called silicide. The silicide-poly-silicon combination has better electrical properties than poly-silicon alone and still does not melt in subsequent processing. Also the threshold voltage is not significantly higher than with poly-silicon alone, because the silicide material is not near the channel [25]. From the $45 \mathrm{~nm}$ node onwards, the metal gate technology returns, together with the use of high-dielectric (high-k) materials, pioneered by Intel developments.

One of the problems in the planar MOSFET was the quantum tunneling effect when reducing the insulator (normally $\mathrm{SiO}_{2}$ ). One of the solutions applied in the tri-gate manufacturing was using high-k material other than $\mathrm{SiO}_{2}$ whose $k=3.9$ but using high-k materials with 
poly-Si had two effects. Firstly, high-k dielectrics and poly-Si are incompatible due to the Fermi level pinning at the poly-Si/high-k interface [26], which causes high threshold voltages in MOSFET transistors. Secondly, the coupling of low surface optical phonon modes, which results from the polarization of high-k dielectric, reduces the electron mobility [27]. This was partly solved by metal/high-k junction at the gate as the metal prevents the coupling of the phonons to the channel under certain conditions improving the mobility [28, 29] like Hafnium dioxide $-\mathrm{HfO}_{2}$ - high-k/TiN metal gate but still the problem of high threshold voltage, also it requires definite type of $n$-channel and $p$-channel materials of certain work function for high performance CMOS logic [30]. Now Intel has engineered n-type and p-type metal electrodes that have the correct work functions on the high- $\mathrm{K}$ for high-performance CMOS [27, 31].

\subsection{Fabrication Process Overview}

In general, both planar and multi-gate transistors share same fabrication processes, but the main difference is how these steps are applied to produce a specific device with its specifications, like length, width, etc. The process steps for devices fabrication will be briefly explained, and then there will be a focus on the main types of multi-gate transistor.

\subsubsection{Planar MOSFET}

Device fabrication process has some major steps that are generally needed to manufacture a transistor device, whether planar or 3D multi-gate; but in multi-gate, these steps are extended much more to achieve the new design of it. Starting by the planar MOS transistor fabrication, detailed process steps are shown in Fig. 6 $[32,33]$.

The process starts with a silicon substrate that faces thermal oxidation to end up with a thin $\mathrm{SiO}_{2}$ layer. The entire oxide surface is covered with a layer of a photo-resistive material, essentially light sensitive called photoresist. The photoresist is initially insoluble during the development (Figs. 6a-b).
The photoresist is then exposed to ultraviolet light. The exposed areas become soluble and are no longer resisting the etching chemicals or etching solvents. To control the soluble areas over the surface, masks are used. Those masks cover some areas of the photoresist and leave other areas uncovered. Thus, when the mask on top is exposed to UV light, areas which are covered by the opaque features on the mask are shielded and insoluble, while other uncovered areas (which UV passes through) become soluble (Fig. 6c).

There are two types of photoresists: positive and negative photoresists. Positive photoresists are initially insoluble and become soluble after UV exposure. Negative photoresists are initially soluble and become insoluble after UV exposure. Negative photoresists are more sensitive to light, but their photolithographic resolution is not as high as that of the positive photoresists. Therefore, negative photoresists are used less commonly in the manufacturing of high-density integrated circuits.

After the exposure step, the exposed areas of the photoresist can be easily removed by a solvent (chemical reaction happens), then the silicon oxide areas that are not covered by the insoluble/hardened photoresist can be etched (removed) by a chemical solvent, such as Hydrofluoric acid. After etching the silicon dioxide by a solvent, an access called "window" becomes available directly to the silicon substrate (Figs. 6d-e).

The remaining photo-resistive areas can be easily stripped by another solvent leaving the rest of the patterned silicon oxide feature over the silicon substrate (Fig. 6f).

Then, the resulting surface is covered by a thin high quality oxide. This oxide will eventually form the gate oxide (insulator) for this MOS transistor. After that, another layer of poly-silicon (metal) is used on top of the previous Gate thin high quality oxide (Figs. 6g-h), then the gate electrode is made according to the desired specifications and dimensions, so the poly-silicon is patterned and etched away in the sub-sequence of steps shown in Figs. 6i-1. 


\section{(a)}

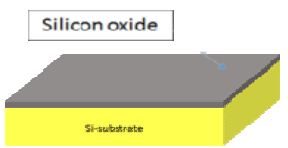

(d) (b)

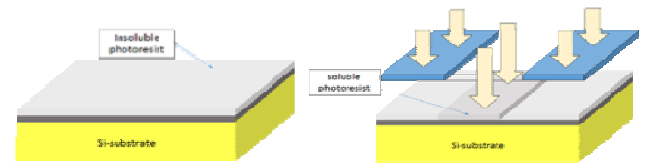

(e)

(f)

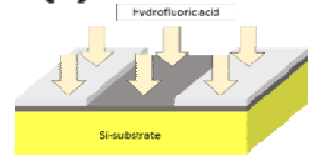

(g)

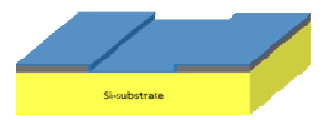

(i)

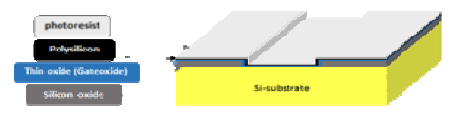

(k)

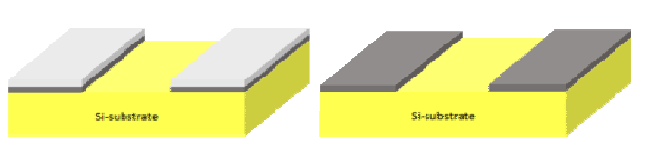

(h)
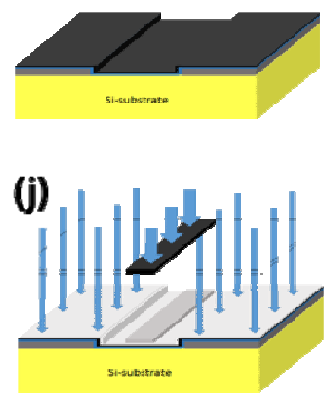

(I)

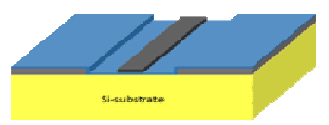

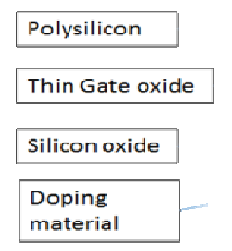

(m)

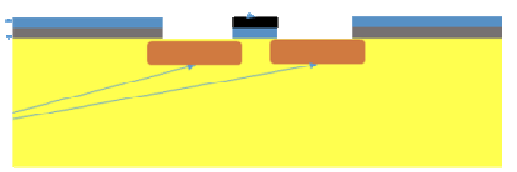

(n)

(o)

(p)

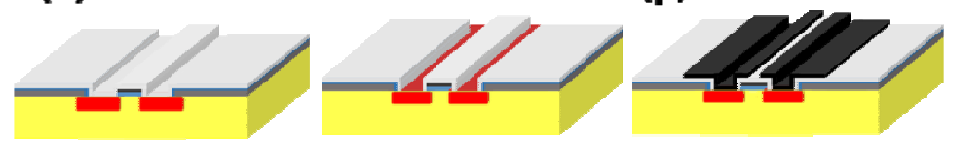

Fig. 6 Fabrication processes of planar transistor.

Afterwards, the area of the thin gate oxide not covered by the poly-silicon is removed and etched away, giving us a direct clean access to the bare silicon substrate where the doping will take place. After etching the thin gate oxide around the area uncovered by the poly-silicon, the entire surface is highly doped with Si-substrate opposite-type concentration impurities. There are two ways of doping: diffusion or ion-implantation.
In diffusion, the unprotected part of the wafer is exposed to an intensive source of opposite-type impurity. Ion implantation accelerates the impurity ions to a very high level then implants the ions into the piece of wafer, in other words, a beam of opposite-type dopant impurity is to be shot into the wafer. The results of such doping here are the most important terminals of the MOS transistor; the source and the drain, colored in orange in Fig. $12 \mathrm{~m}$. 
After forming the correct desired source and drain specifications, the whole surface is covered by an insulating layer of silicon dioxide. Then, the oxide layer is patterned and etched -using UV and lithography as before- to form connecting windows to the source and the drain to form the MOSFET circuitry (Figs. 6n and 6o).

Finally, the surface is covered with evaporated aluminum to form the device interconnects and metal windows, then the aluminum surface is patterned and etched to complete the fabrication process of this planar MOSFET (Fig. 6p).

\subsubsection{Tri-gate FET}

The fabrication process of multi-gate FETs and the planar MOSFETs are somehow similar in general. Below is the explanation of some types of Multi-Gate Transistor. Starting by the tri-gate Transistor, the SOI (Silicon-On-Insulator) configuration is usually begun with to reduce the parasitic capacitances. Sometimes, SOS (Silicon-On-Sapphire) is used but this happens mostly in electronic devices with RF constrains.
Insulator layers are used at the beginning as shown in Figs. 7a-d. Beginning with Silicon on insulator structure is considered a step that is commonly used in all kinds of Multi-Gate FETs. Now coating with a positive photo-resistive layer takes place and applying UV exposure, then chemically removing the solvable areas resultant of the lithography UV exposure.

Afterwards, another chemical solvent is applied to the surface of the wafer to etch the areas not covered by the photoresist, and then etch the rest of the photoresist. So this stage ends with the structure shown in Fig. 7e.

Then coating the previous structure with poly-silicon takes place to form the gate electrode, passing through a planarization step, and ending with what is shown in Fig. 7f.

Now a photoresist layer is applied, then lithographing is masked over it, and chemically the solvent is removed by a reactant that only reacts with the unprotected layer of gate electrode. The result is a SOI Tri-Gate FET. Afterwards, some etching takes place to remove the areas uncovered by the insoluble
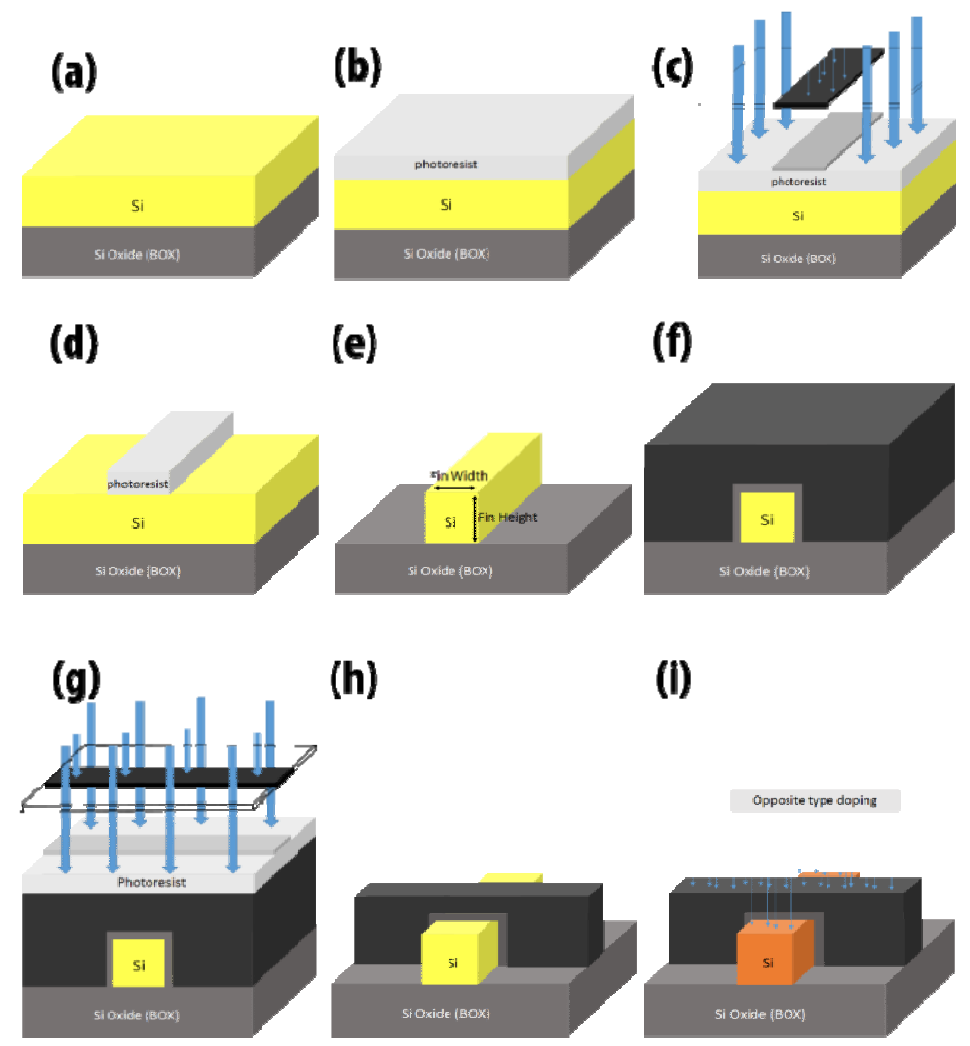

(h)

(i)

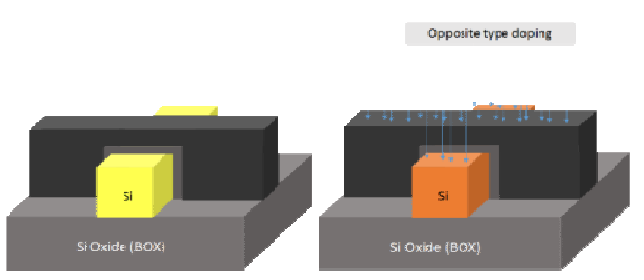

Fig. 7 Fabrication processes of tri-gate transistor. 
photoresist after the UV exposure, and then the remaining of the photoresist itself is etched, which was covering the gate area, ending with the structure shown in Figs. 7g-h.

Finally, doping process takes place to shape the source and drain fins as shown in Fig. $7 \mathrm{i}$.

\subsubsection{Double-Gate FET}

A simple SOI structure begins this process also (Fig. 8a). The whole surface is covered with a photoresist, which is preferred to be positive. Exposure with the desired mask that matches device dimensions takes place, then etching the soluble photoresist then the underlying silicon (areas uncovered), then etching the unneeded photoresist that was not exposed. This is shown in Figs. 8b-c.

The whole surface is covered again with silicon doped whether $\mathrm{P}$ or $\mathrm{N}$ to form the fins for source and drain in Fig. 8d. The new photo-resistive area is exposed and then the resultant areas not covered by photoresist are etched (Fig. 8e), then the rest of the photoresist is removed (Fig. 8f).

The surface is covered with poly-silicon to form the gate electrode then it is patterned and etched. The doping is made with opposite type material to form the final double gate FET as in Fig. 8g.

\subsection{4 $\Omega$-Gate FET}

Starting from the stage of the double-gate FET previously explained, where we have patterned, etched away and reached out to the structure shown in Figs. $9 \mathrm{a}-\mathrm{c}$, then coated the surface with poly-silicon to form the gate electrode and patterning it with a mask -having features of the desired gate length- passing through etching away the soluble photoresist -gate electrode area not covered by it- and finally etching the rest insoluble photoresist.

Again, we will cover the whole surface with positive
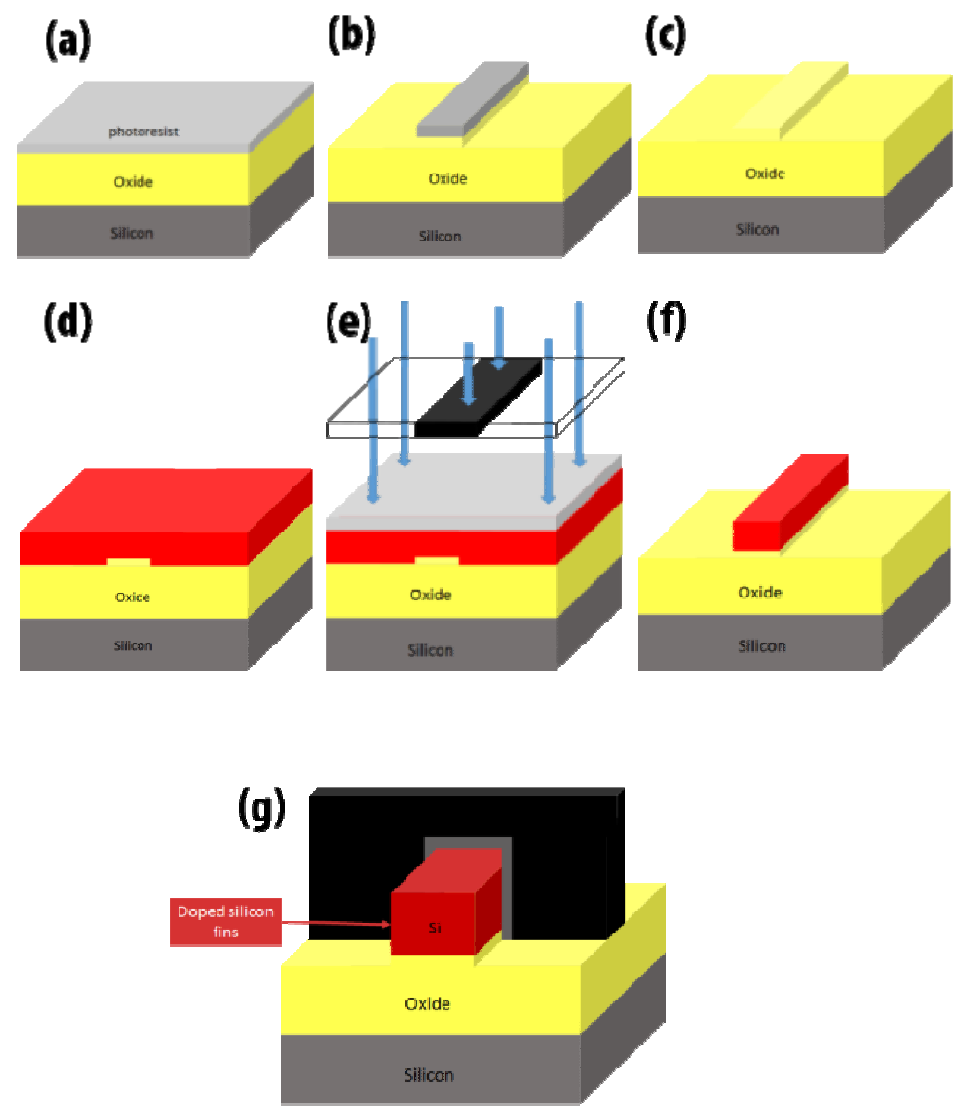

Fig. 8 Fabrication processes of double gate transistor. 


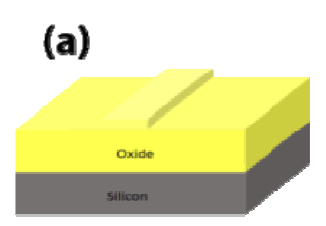

(b)

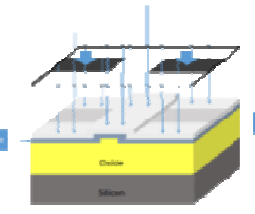

(e)
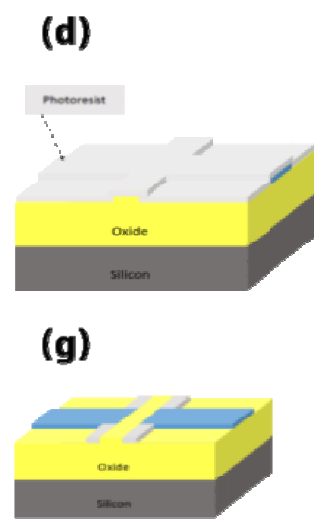

(j)

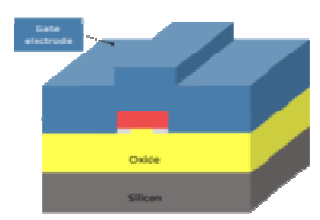

(h)
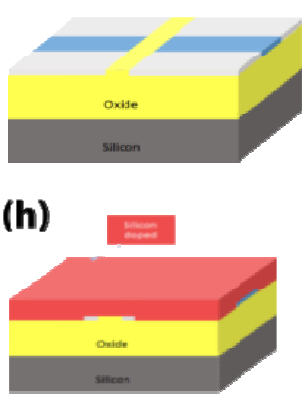

(k)

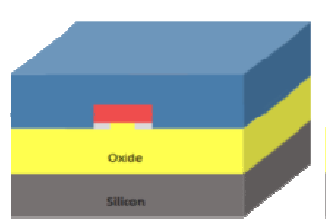

(m)

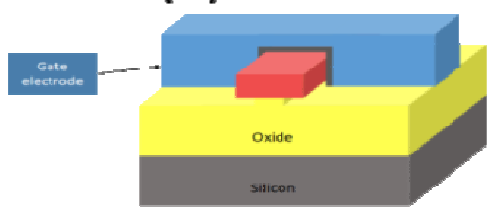

Fig. 9 Fabrication processes of gate transistor. photoresist, then remove the photoresist stack using planarization techniques. CMP (chemical mechanical planarization) shown in Figs. 9d-e is a famous planarization method in semiconductor devices fabrication.

Patterning process then takes place to result in the structure shown in Figs. 9f-g, the resultant photoresist areas work along with the oxide bar between them as fins' basements. These areas will be removed in the final step.

A silicon doped material is then coated above the surface then we apply planarization to the surface to flatten it. Patterning and etching the silicon surface processes take place after planarization to create the fins, the source and the drain. The fin is resting on both silicon oxide and the photoresist parts as shown in Figs. (c)

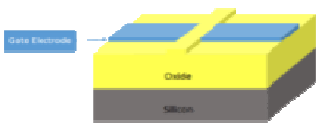

(f)
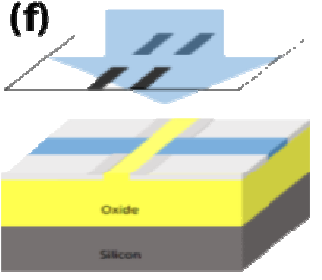

(i)

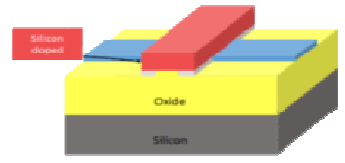

(I)

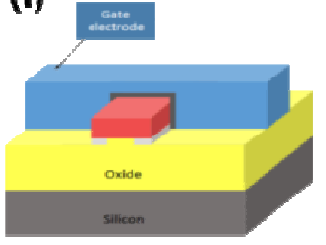

9h and $9 \mathrm{i}$.

Now we have to cover the surface with poly-silicon to create the rest of the gate electrode over the other sides of the fins. CMP is used to platen the gate electrode surface to make it ready for exposure.

The gate electrode now is formed around the three sides of the fins and the photoresist parts still exist as shown in Figs. 9j-1.

Finally, remove the insoluble photo-resistive parts by chemical etching to end up with the final device shown in Fig. $9 \mathrm{~m}$ ready for operation as $\Omega$-gate FinFET.

\section{Conclusions}

In this paper, a detailed review on the different categories of 3D Multi-gate transistors was introduced, 
including the structure, operation, and fabrication. On account of the continuous advancement of VLSI designs and architectures, and the pursuit of more speed and ultra-low power electronics chips, the traditional planar MOSFET device became the bottleneck of the growing demand for larger Systems on Chip. The drawback of the planar transistors was illustrated, like the short channel effect when decreasing the channel length of the device. The idea of Multi-gate FETs became the ideal solution to the negative effects of channel decreasing of planar MOSFET. The different structures and their fabrication techniques of Multi-gate devices provide chip makers with devices with small channel length, and faster switching efficient speed operation, and lower power consumption, due to the much better control over the device channel. Hereunder, it solved the problems of short channel effects, and gave us a chance to decrease the device size, and optimize the usage of die size in chip fabrication, or even give designer the promise for more functionality over same chip size.

\section{References}

[1] Ferain, I., Colinge, C. A., and Colinge, J. P. 2011. "Multigate Transistors as the Future of Classical Metal-Oxide-Semiconductor Field-Effect Transistors." Nature 479 (7373): 310-6.

[2] Razavi, B. 2008. Fundamentals of Microelectronics: BR Wiley.

[3] Bhole, M., Kurude, A., and Pawar, S. 2013. "3D Tri-gate Transistor Technology and Next Generation FPGAs." International Journal of Engineering Sciences and Research Technology 2: 2670-5.

[4] Rabaey, J. M., Chandrakasan, A. P., and Nikolic, B. 2002. Digital Integrated Circuits, Vol. 2: Prentice Hall Englewood Cliffs.

[5] Colinge, J. P. 2013. “3D Transistors.” Presented at 2013 International Symposium on VLSI Technology, Systems, and Applications (VLSI-TSA), Hsinchu, Taiwan.

[6] Conde, A. O., S'anchez, F. J. G., Liou, J. J., Cerdeira, A., Estrada, M., and Yue, Y. 2002. "A Review of Recent Mosfet Threshold Voltage Extraction Methods." Microelectronics Reliability 42 (4): 583-96.

[7] Singhal, S., Kumar, S., Upadhyay, S., and Nagaria, R. K. 2013. "Comparative Study of Double Gate SOI Finfet and Trigate Bulk MOSFET Structures.” Presented at 2013
Students Conference on Engineering and Systems (SCES), Allahabad, India.

[8] Tsividis, Y. 1999. Operation and Modeling of the MOS Transistor: Oxford University Press.

[9] Park, J. T., and Colinge, J. 2002. "Multiple-Gate SOI MOSFETS: Device Design Guidelines." IEEE Transactions on Electron Devices 49 (12): 2222-9.

[10] Park, J. T., Colinge, J., and Diaz C. H. 2001. "Pi-Gate SOI MOSFET." IEEE Electron Device Letters 22 (8): 405-6.

[11] D'Agostino, F., and Quercia, D., Short-Channel Effects in MOSFETs. Introduction to VLSI Design (EECS 467), 2000.

[12] Zeghbroeck, B. V. 2004. Principles of Semiconductor Devices. Colarado University.

[13] Yan, R. H., Ourmazd, A., and Lee, K. F. 1992. "Scaling the Si MOSFET: From Bulk to SOI to Bulk." IEEE Transactions on Electron Devices 39 (7): 1704-10.

[14] Kuhn, K. J. 2012. "Considerations for Ultimate CMOS Scaling.” IEEE Trans. Electron Devices 59 (7): 1813-28.

[15] Colinge, J. P. 2007. "Quantum-Wire Effects in Trigate SOI MOSFETS.” Solid-State Electronics 51 (9): 1153-60.

[16] Colinge, J. P. 2014. "Multigate Transistors: Pushing Moore's Law to the Limit." Presented at 2014 International Conference on Simulation of Semiconductor Processes and Devices (SISPAD), Yokohama, Japan.

[17] Balestra, F., Cristoloveanu, S., Benachir, M., Brini, J., and Elewa, T. 1987. "Double-Gate Silicon-Oninsulator Transistor with Volume Inversion: A New Device with Greatly Enhanced Performance." IEEE Electron Device Letters 8 (9): 410-2.

[18] Colinge, J. P. 2008. "The New Generation of SOI MOSFETS.” Romaniam Journal Information Science and Technology 11 (1): 3-15.

[19] Colinge, J., Quinn A. J., Floyd, L., Redmond, G., Alderman, J. C., Xiong, W., Cleavelin, C. R., Schulz, T., Schruefer, K., Knoblinger, G. et al. 2006. "Low-Temperature Electron Mobility in Tri-gate SOI MOSFETs." IEEE Electron Device Letters 27 (2): 120-2.

[20] COMSOL Multiphysics Software Site [Online]. Available: www.comsol.com.

[21] Srivastava, V. M., and Singh, S. P. 2012. "Analysis and Design of Tri-gate MOSFET with High Dielectrics Gate." International Journal of Intelligent Systems and Applications 4 (5): 16.

[22] Kumar, V., and Agarwal, S. K. 1998. Physics of Semiconductor Devices. Vol. 1, Narosa.

[23] Baliga, B. J. 2010. Fundamentals of Power Semiconductor Devices. Springer Science \& Business Media.

[24] Long, R. D., and McIntyre, P. C. 2012. "Surface Preparation and Deposited Gate Oxides for Gallium 
Nitride Based Metal Oxide Semiconductor Devices." Materials 5 (7): 1297-335.

[25] Vasileska, D., and Goodnick, S. M. 2005. "Computational Electronics." Synthesis Lectures on Computational Electromagnetics, 1 (1):1-216.

[26] Hobbs, C., Fonseca, L., Knizhnik, A., Dhandapani, V., Samavedam, S. B., Taylor, W. J., Grant, J. M., Dip, L. G., Triyoso, D. H., Hegde, R. I., Gilmer, D. C. , Garcia, R., Roan, D., Lovejoy, M. L., Rai, R. S., Hebert, E. A., Tseng, H. H., Anderson, S. H., White, B. E., and Tobin, P. J. 2003. "Fermi Level Pinning at the Poly-Si/Metal Oxide Interface.” In Proceedings of 2003 Symp. VLSI Tech. Dig, 9-10.

[27] Chau, R. "Advanced Metal Gate/High-K Dielectric Stacks For High-Performance CMOS Transistors." Logic Technology Development, Intel Corporation.

[28] Fischetti, M. V., Neumayer, D. A., and Cartier, E. A. 2001. "Effective Electron Mobility in Si Inversion Layers in Metal-Oxide-Semiconductor Systems with a High-Insulator: The Role of Remote Phonon Scattering." Journal of Applied Physics 90 (9): 4587-608.
[29] Doyle, B., Boyanov, B., Datta, S., Doczy, M., Hareland, S., Jin, B., Kavalieros, J., Linton, T., Rios, R., and Chau, R. 2003. "Tri-gate Fully-Depleted CMOS Transistors: Fabrication, Design and Layout." In Proceedings of 2003 Symposium on VLSI Technology, 133-4.

[30] Lu, Q., Lin, R., Ranade, P., King, T. J., and Hu, C. 2001. "Metal Gate Work Function Adjustment for Future CMOS Technology." In Proceedings of 2001 Symposium on VLSI Technology, 45-6.

[31] Cheng, K., Khakifirooz, A., Kulkarni, P., Ponoth, S., Kuss, J., Shahrjerdi, D., Edge, L. F., Kimball, A., Kanakasabapathy, S., Xiu, K., et al. 2009. "Extremely Thin SOI (ETSOI) CMOS with Record Low Variability for Low Power System-On-Chip Applications.” Presented at 2009 IEEE International Electron Devices Meeting (IEDM), Baltimore, USA.

[32] Kang, S. M., and Leblebici, Y. 2002. CMOS Digital Integrated Circuits Analysis \& Design. New York: McGraw-Hill, Inc.

[33] Colinge, J. P., et al. 2008. FinFETs and Other Multi-gate Transistors. Springer. 